\title{
Using SPM to Detect Evolving MS Lesions
}

David Rey, Jonathan Stoeckel, Grégoire Malandain, and Nicholas Ayache

\author{
INRIA Sophia - Epidaure Project \\ 2004 Route des Lucioles BP 93 \\ 06902 Sophia Antipolis Cedex, France \\ \{David.Rey, Jonathan.Stoeckel, Gregoire.Malandain, \\ Nicholas. Ayache\}@sophia.inria.fr \\ http://www-sop.inria.fr/epidaure/Epidaure-eng.html
}

\section{Introduction}

Clinicians need to study the effects of new treatments: it is sometimes possible to detect and quantify those effects by looking at evolutions in the medical images of a patient over time especially in the case of multiple sclerosis (MS) where lesions are related to clinical signs [1]. Some methods allow to compare two images to know where there are differences, typically between the last and the previous exam [2] 3]. However a retrospective analysis might be done on the whole set of images to find the moments when evolutions occur [4. We propose to use the analogy between an activation in functional imaging (for instance PET, SPECT and fMRI) [5] and a signal change due to an evolving multiple sclerosis lesion. Voxels corresponding to evolving pathological areas are named ELV (Evolving Lesion Voxels) in this abstract.

\section{Method}

First of all we have to pre-process the image series: geometrical alignment [6] and temporal bias correction using the joint histogram of two images.

Considering the shape of ELV profiles over time (Fig. 1 -a), we use a kind of asymmetric Gaussian (Fig. 1 -b) with five parameters: amplitude, mean, rising time, decreasing time and vertical offset, $f(x)=p_{1} \times \exp \left(-g\left(x-p_{2}\right)^{2}\right)+p_{5}$ with $g(x)=p_{3} \times\left(x+\sqrt{1+x^{2}}\right)+p_{4} \times\left(x-\sqrt{1+x^{2}}\right)$. This model is fitted on a training set of ELV profiles and normalized to obtain an average model of evolving lesions (Fig. 1 1 c). The analysis is computed independently for each time point, thus resulting in as many analyses as the number of temporal images. Those separate analyses are due to the necessity to have enough data to preserve sensitivity. For each analysis, first the best linear combination of the average model and a constant value is estimated for each voxel (Fig. 2). Then we proceed to the statistical inference stage (using a $t$-test).

Thus our method takes into account both temporal and spatial cohesion of the evolving pathological areas: the temporal cohesion is due to the use of a temporal signal model of voxels in evolving lesions, and the spatial cohesion comes from the statistical analysis method that makes it possible to compute probabilities for clusters of voxels. 
(a)

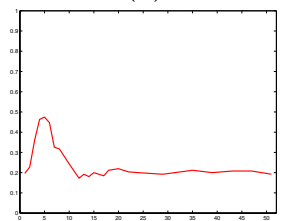

(b)

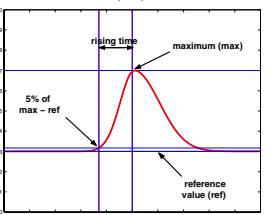

(c)

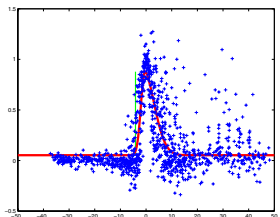

Fig. 1. a) typical curves of evolving pathological voxel over time are composed of a rising part and a decreasing part. b) An instance of our model of evolving profile with five parameters. c) Normalized training set of profiles with the fitted. average model

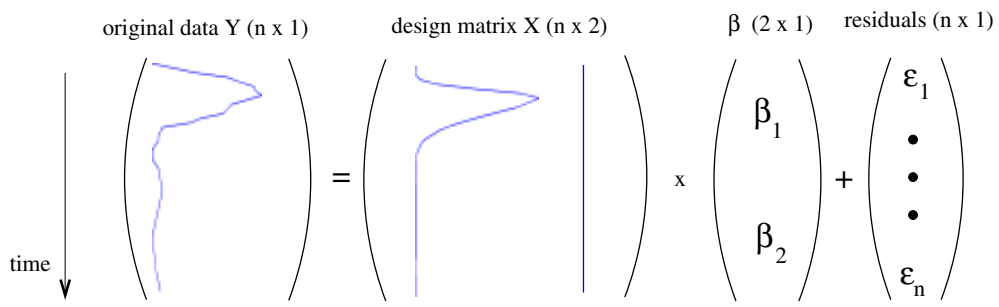

Fig. 2. General linear model

The described statistical analysis was applied on a time series of $24 \mathrm{~T} 2$-weighted MRI acquired over one year 1 . Each image has a size of $256 \times$ $256 \times 54$ with a voxel size of $0.9375 \times 0.9375 \times 3.0 \mathrm{~mm}^{3}$. We used the freely available SPM99 packag 2 for the statistical analysis. We only kept Fig. 3. It is possible to superimpose evolving arthe clusters which sizes have a probability smaller than 0.01 .

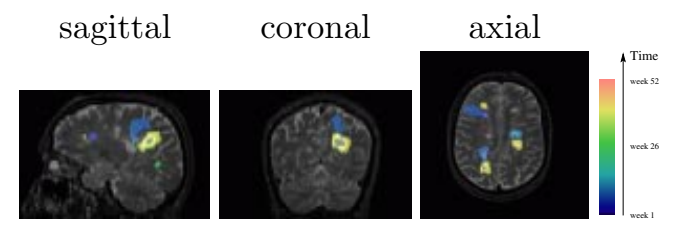

This new approach for detecting MS lesions combines temporal (model of ELV profiles) and spatial cohesion (SPM framework) providing promising results.

\section{References}

[1] C. R. G. Guttmann, S. S. Ahn, L. Hsu, R. Kikinis, and F. A. Jolesz. The Evolution of Multiple Sclerosis Lesions on Serial MR. American Journal of NeuroRadiology, 16:1481-1491, August 1995.

[2] D. Rey, G. Subsol, H. Delingette, and N. Ayache. Automatic Detection and Segmentation of Evolving Processes in 3D Medical Images: Application to Multiple Sclerosis. In IPMI'99, volume 1613 of LNCS, pages 154-167, June 1999.

${ }^{1}$ courtesy of Dr. Charles Guttmann and Dr. Ron Kikinis, Brigham and Women's Hospital, and Harvard Medical School

${ }^{2}$ http://www.fil.ion.ucl.ac.uk/spm 
[3] D. Rey, C. Lebrun-Frénay, G. Malandain, S. Chanalet, N. Ayache, and M. Chatel. A New Method to Detect and Quantify Evolving MS Lesions by Mathematical Operators. In $A A N$, volume 54 of Neurology (Supp. 3), page 123, May 2000.

[4] G. Gerig, D. Welti, C. Guttmann, A. Colchester, and G. Székely. Exploring the discrimination power of the time domain for segmentation and characterization of active lesions in serial MR data. Medical Image Analysis, 4(1):31-42, 2000.

[5] R.S.J. Frackowiak, K.J. Friston, C.D. Frith, R.J. Dolan, and J.C. Mazziotta. Human Brain Function. Academic Press, 1997.

[6] J. P. Thirion. New Feature Points Based on Geometric Invariants for 3D Image Registration. International Journal of Computer Vision, 18(2):121-137, May 1996. 